\title{
Téoros
}

Revue de recherche en tourisme

\section{Les stars au firmament des destinations}

\section{Michèle Laliberté}

Volume 24, numéro 1, printemps 2005

URI : https://id.erudit.org/iderudit/1071114ar

DOI : https://doi.org/10.7202/1071114ar

Aller au sommaire du numéro

Éditeur(s)

Université du Québec à Montréal

ISSN

0712-8657 (imprimé)

1923-2705 (numérique)

Découvrir la revue

Citer cette note

Laliberté, M. (2005). Les stars au firmament des destinations. Téoros, 24(1),

72-74. https://doi.org/10.7202/1071114ar d'utilisation que vous pouvez consulter en ligne.

https://apropos.erudit.org/fr/usagers/politique-dutilisation/ 


\section{Les stars au firmament des destinations}

\section{Michèle Laliberté}

Plusieurs destinations gagnent en popularité, deviennent plus accessibles et voient croître substantiellement leurs arrivées touristiques, l'espace de quelques années. Ces protagonistes érodent les parts de marché des destinations classiques et menacent d'en déloger certaines du grand classement mondial. Comment se profile la concurrence du Canada sur l'échiquier international ${ }^{1}$ ?

\section{Une année 2004 sous le signe de la reprise}

Après trois années difficiles, l'année 2004 revient en force avec la plus importante croissance des arrivées touristiques internationales depuis dix ans. L'Organisation mondiale du tourisme (OMT) estime à 760 millions le volume des arrivées, soit une hausse de $10 \%$ par rapport à 2003.

\section{L'Europe des grands classiques et des vedettes montantes}

La France, l'Espagne, l'Italie, le Royaume-Uni, l'Autriche et l'Allemagne demeurent les grands classiques européens. Bien que ces pays n'enregistrent pas de taux de croissance spectaculaires et subissent parfois des baisses, ils figurent toujours parmi les dix plus grands de la planète. Dans cette même région du globe, la Turquie, la Croatie et la Russie sont considérées comme les vedettes montantes.

\section{Tableau 1}

Variation des arrivées internationales des pays en forte croissance Région Europe

\begin{tabular}{l|c|c|c|c|c} 
EUROPE & $\begin{array}{c}\mathbf{0 0 / 9 9} \\
\mathbf{\%} \\
\text { variation }\end{array}$ & $\begin{array}{c}\mathbf{0 1 / 0 0} \\
\mathbf{\%} \\
\text { variation }\end{array}$ & $\begin{array}{c}\mathbf{0 2 / 0 1} \\
\mathbf{\%} \\
\text { variation }\end{array}$ & $\begin{array}{c}\mathbf{0 3 / 0 2} \\
\mathbf{\%} \\
\text { variation }\end{array}$ & $\begin{array}{c}\mathbf{2 0 0 3} \\
\text { Arrivées } \\
\text { internationales } \\
\text { (milliers) }\end{array}$ \\
\hline Turquie & 39,1 & 12,5 & 18,6 & 4,3 & 13341 \\
Russie & 14,5 & 5,3 & 7,3 & 0,9 & 8015 \\
Croatie & $\mathrm{n} / \mathrm{a}$ & 12,2 & 6,1 & 6,7 & 7409 \\
\hline
\end{tabular}

Source: Données tirées de l'Organisation mondiale du tourisme, 2001, 2003 et 2004.

Après une année 1999 difficile en raison d'un violent tremblement de terre, la Turquie a repris son ascension. L'année 2004 s'annonce une autre année record alors que le ministère de la Culture et du Tourisme turque estime à 17,5 millions le nombre de voyageurs étran- gers. De quoi rivaliser avec le Canada pour une position au classement du plus grand nombre d'arrivées internationales!

Une vitesse de croisière se profile pour la Fédération de Russie. Après le boom de 1999 et 2000, son ascension s'est poursuivie avec plus de 8 millions de visiteurs en 2003.

La Croatie est devenue la nouvelle coqueluche de l'Europe et les touristes qui la fréquentent y dépensent de plus en plus. En 2004, les chiffres provisoires diffusés par le pays font état d'une augmentation de 19\% des touristes internationaux, ce qui porte leur nombre à 8,8 millions.

\section{Les Caraïbes et l'Amérique latine redorent la performance des Amériques}

Les États-Unis représentent la destination phare de ce continent. Quant au Canada, il bataille surtout contre le Mexique pour obtenir une plus grande part de marché.

Tableau 2

Variation des arrivées internationales des pays en forte croissance Région des Amériques

\begin{tabular}{l|c|c|c|c|c}
\hline AMÉRIQUES & $\begin{array}{c}\mathbf{0 0 / 9 9} \\
\mathbf{\%} \\
\text { variation }\end{array}$ & $\begin{array}{c}\mathbf{0 1 / 0 0} \\
\mathbf{\%} \\
\text { variation }\end{array}$ & $\begin{array}{c}\mathbf{0 2 / 0 1} \\
\mathbf{\%} \\
\text { variation }\end{array}$ & $\begin{array}{c}\mathbf{0 3 / 0 2} \\
\mathbf{\%} \\
\text { variation }\end{array}$ & $\begin{array}{c}\mathbf{2 0 0 3} \\
\text { Arrivées } \\
\text { internationales } \\
\text { (milliers) }\end{array}$ \\
\hline Brésil & 4,0 & $-10,2$ & $-20,7$ & 8,1 & 4091 \\
$\begin{array}{l}\text { République } \\
\text { dominicaine }\end{array}$ & 12,4 & $-3,0$ & $-2,5$ & 16,8 & 3282 \\
Argentine & 3,2 & $-9,9$ & 7,6 & 6,2 & 2995 \\
Cuba & 8,9 & $-0,3$ & $-4,6$ & 11,5 & 1847 \\
Chili & 7,4 & $-1,1$ & $-18,0$ & 14,3 & 1614 \\
Costa Rica & $\mathrm{n} / \mathrm{a}$ & 4,0 & $-1,6$ & 11,3 & 1239 \\
Pérou & $\mathrm{n} / \mathrm{a}$ & $\mathrm{n} / \mathrm{a}$ & 8,2 & 8,0 & 931 \\
\hline
\end{tabular}

Source : Données tirées de l'Organisation mondiale du tourisme, 2001, 2003 et 2004.

Les années 2001 et 2002 se sont soldées globalement par un bilan négatif pour l'ensemble des Amériques. Malgré cela, la République dominicaine et Cuba ont le vent dans les voiles. Ces pays ont enregistré des taux de croissance de leurs arrivées variant de 8,9 à 16,8 \% depuis 1999 et, pendant les deux années sombres, la diminution ne s'est pas révélée aussi importante que dans plusieurs autres destinations. 
L'année 2003 a sonné le coup d'envoi pour plusieurs pays d'Amérique latine, soit le Brésil, l'Argentine, le Chili, le Costa Rica et le Pérou.

\section{L'Asie-Pacifique, I'enfant prodige}

Bien que le syndrome respiratoire aigu sévère (SRAS) soit venu interrompre son ascension en 2003, l'étoile qui brille au firmament des destinations est sans contredit la région Asie-Pacifique.

Tableau 3

Variation des arrivées internationales des pays en forte croissance Région Asie/Océanie

\begin{tabular}{l|c|c|c|c|c} 
ASIE / OCÉANIE & $\begin{array}{c}\mathbf{0 0 / 9 9} \\
\mathbf{\%} \\
\text { variation }\end{array}$ & $\begin{array}{c}\mathbf{0 1 / 0 0} \\
\mathbf{\%} \\
\text { variation }\end{array}$ & $\begin{array}{c}\mathbf{0 2 / 0 1} \\
\mathbf{\%} \\
\text { variation }\end{array}$ & $\begin{array}{c}\mathbf{0 3 / 0 2} \\
\mathbf{\%} \\
\text { variation }\end{array}$ & $\begin{array}{c}\mathbf{2 0 0 3} \\
\text { Arrivées internationales } \\
\text { (milliers) }\end{array}$ \\
\hline Chine & 15,5 & 6,2 & 11,0 & $-10,4$ & 32970 \\
Hong Kong (Chine) & 15,3 & 5,1 & 20,7 & $-6,2$ & 15537 \\
Malaisie & 28,9 & 25,0 & 4,0 & $-20,4$ & 10577 \\
Thaïlande & 9,9 & 5,8 & 7,3 & $-7,3$ & 10082 \\
Macao (Chine) & 32,3 & 12,4 & 12,4 & $-3,9$ & 6309 \\
Japon & 7,2 & 0,3 & 9,8 & $-0,5$ & 5212 \\
Inde & $n / a$ & $-4,2$ & $-6,0$ & 15,4 & 2750 \\
Nouvelle-Zélande & $n / a$ & 6,9 & 7,1 & 2,9 & 2104 \\
\hline
\end{tabular}

Source: Données tirées de l'Organisation mondiale du tourisme, 2001, 2003 et 2004.

Le potentiel de croissance économique, l'importance de la population et la libéralisation des voyages propulseront la Chine comme LA destination mondiale et peut-être plus tôt que prévu. Elle est passée du treizième rang des arrivées touristiques internationales en 1985 au cinquième en 2003, exploit qu'aucune autre destination n'a réussi à accomplir.

Avec des hausses spectaculaires des arrivées internationales, la Malaisie se hisse parmi les grands de cette région du globe et surpasse même la Thailande, une autre vedette montante.

Malgré un volume de visiteurs moindre, le Japon a obtenu des taux de croissance intéressants trois années sur quatre et n'a connu qu'une très faible diminution lors du SRAS.

L'Inde est à surveiller du coin de l'œil. Même s'il ne représente que 2,7 millions de touristes étrangers, ce pays a tout même enregistré une majoration de 15,4 \% de ses arrivées en 2003 et celle de 2004 serait supérieure. À l'instar de la Chine, ses perspectives de croissance sont grandes et le gouvernement indien a bien l'intention de dynamiser ce secteur.

Malgré la baisse généralisée en Asie-Pacifique à la suite du SRAS, la Nouvelle-Zélande et l'Inde ont été les seuls pays à afficher une croissance positive des arrivées en 2003. Avec l'effet combiné du film Le Seigneur des anneaux et de son plan marketing efficace, la Nouvelle-Zélande poursuit sa lancée.

\section{Le Moyen-Orient et ses projets de démesure}

Les données préliminaires de 2004 font état d'une croissance de $20 \%$ des touristes pour le Moyen-Orient. II se hisse ainsi au quatrième rang des régions les plus visitées devant l'Afrique. L'Arabie saoudite représente la principale destination de cette partie du globe avec 7,3 millions d'arrivées en 2003 et certains pays progressent de façon notable.

Exception faite de l'année 2001, l'Égypte continue son ascension avec des taux de croissance supérieurs à $12 \%$ depuis les dernières années. Le Liban et la Jordanie tirent bien leur épingle du jeu malgré des variations plus inégales de leurs arrivées.

Les Émirats arabes unis, Dubaï en tête, et le Bahreïn se taillent progressivement une place au sein de cette région. II faut souligner les mégaprojets touristiques de Dubaï et son ambitieux objectif de tripler le nombre de touristes étrangers d'ici 2010.

\section{Une progression de plusieurs pays du continent africain}

En 2003, l'Afrique du Sud arrive en tête des arrivées internationales (6,6 millions) du continent africain.

\section{Tableau 4}

Variation des arrivées internationales des pays en forte croissance Région du Moyen-Orient / Afrique

\begin{tabular}{l|c|c|c|c|c}
\hline MOYEN-ORIENT & $\begin{array}{c}\mathbf{0 0 / 9 9} \\
\mathbf{\%} \\
\text { variation }\end{array}$ & $\begin{array}{c}\mathbf{0 1 / 0 0} \\
\mathbf{\%} \\
\text { variation }\end{array}$ & $\begin{array}{c}\mathbf{0 2 / 0 1} \\
\mathbf{\%} \\
\text { variation }\end{array}$ & $\begin{array}{c}\mathbf{0 3 / 0 2} \\
\mathbf{\%} \\
\text { variation }\end{array}$ & $\begin{array}{c}\mathbf{2 0 0 3} \\
\text { Arrivées internationales } \\
\text { (milliers) }\end{array}$ \\
\hline $\begin{array}{l}\text { Émirats arabes } \\
\text { unis }\end{array}$ & $\mathrm{n} / \mathrm{a}$ & 5,8 & 31,7 & 7,8 & 5871 \\
Égypte & 14,0 & $-14,8$ & 12,6 & 17,1 & 5746 \\
Bahreïn & $\mathrm{n} / \mathrm{a}$ & 15,2 & 13,6 & $-6,7$ & 2955 \\
Jordanie & 5,1 & 3,6 & 9,8 & $-3,0$ & 1573 \\
Liban & $\mathrm{n} / \mathrm{a}$ & 12,9 & 14,3 & 6,1 & 1015 \\
\hline AFRIQUE & & & & & 5114 \\
Tunisie & 4,7 & 6,5 & $-6,0$ & 1,0 & 4552 \\
Maroc & 7,8 & 6,5 & $-0,9$ & 5,8 & 1166 \\
\hline Algérie & 15,6 & 4,1 & 9,6 & 18,0 & \\
\hline
\end{tabular}

Source: Données tirées de l'Organisation mondiale du tourisme, 2001, 2003 et 2004.

Les pays du Maghreb connaissent de bonnes années et le Maroc vient particulièrement rivaliser avec la Tunisie. Sur la lancée de son plan Azur, les chiffres préliminaires marocains annoncent une augmentation des visiteurs étrangers d'environ $16 \%$ en 2004 pour atteindre 5,5 millions. À moindre échelle, l'Algérie obtient des taux de croissance substantiels depuis cinq ans.

Même si le volume de touristes étrangers se situe sous la barre du million, la Zambie a connu, depuis les cinq dernières années, d'importantes hausses pouvant atteindre jusqu'à $26 \%$. Le Ghana, la Tanzanie et le Kenya ont eux aussi bénéficié, à quelques reprises, d'augmentations avoisinant les $10 \%$. 


\section{Sur un horizon à long terme}

Selon l'OMT, les dix premières destinations constituaient $88 \%$ des arrivées internationales en 1950, proportion qui a diminué à 49,2 \% en 2003 et qui devrait se poursuivre encore pour se situer à 45,9\% en 2020. Le nombre d'arrivées de touristes internationaux aura plus que doublé par rapport à 2003, passant à 1,5 milliard, mais les parts de marché seront modifiées. L'Europe et les Amériques perdront du terrain tandis que l'Asie-Pacifique, l'Afrique et le Moyen-Orient seront en mode croissance. Sur cette lancée, l'Asie-Pacifique surpassera même les Amériques en termes de volumes.

Les destinations qui investissent des sommes importantes dans le tourisme sont légion et plusieurs ont des objectifs ambitieux. À observer la vitesse des changements qui se profilent, le classement des destinations de l'OMT en 2020 pourrait être bousculé.

Serge Trigano, ex-président du Club Méditerranée, exprimait récemment sa vision du tourisme de 2010 en ces termes : "Les pays deviendront de vraies marques, avec leurs personnalités, et se battront à travers de violentes campagnes publicitaires. " Et on peut sûrement lui donner raison.

Et le Canada en $2020 ?$

Michèle Laliberté est analyste au Réseau de veille de la Chaire de Tourisme, École des sciences de la gestion, Université du Québec à Montréal.
Notes

1 Basés sur les données de l'Organisation mondiale du tourisme, les chiffres dans le texte font référence au classement des destinations, aux arrivées et aux recettes touristiques internationales. Afin d'alléger la lecture, certains synonymes sont utilisés. Les données de 2004 sont provisoires et celles de 2003 peuvent ultérieurement être sujettes à modification.

\section{Bibliographie}

« Dix patrons nous livrent leur vision ", L'Écho touristique, 10 décembre 2004 , p. 34, auteur inconnu.

AFP (2005a), « Maroc : le seuil des 5 millions de touristes est franchi en 2004 », Infos Économiques, 3 février.

AFP (2005b), «Le tourisme croate bat tous les records en 2004 ", Tourismexpress, 11 janvier.

Maison de la France (2004), Lettre de veille - Asie du Sud-Est et Inde, $n^{\circ} 2$, septembre.

Office de Tourisme de Turquie - Paris (2005), Hausse de $25 \%$ des arrivées de touristes en Turquie en 2004, [http://www.infosturquie.com], 11 janvier.

Organisation mondiale du tourisme (1999), Tourisme Horizon 2020, Madrid, Espagne.

Organisation mondiale du tourisme (2001), Tourism Highlights, [www.world-tourism.org].

Organisation mondiale du tourisme (2003), Tourism Highlights, [www.world-tourism.org].

Organisation mondiale du tourisme (2004), Tourism Highlights, [www.world-tourism.org].

Organisation mondiale du tourisme (2005), "Le tourisme international obtient ses meilleurs résultats depuis vingt ans », Communiqués de presse, Bangkok, 2 février.

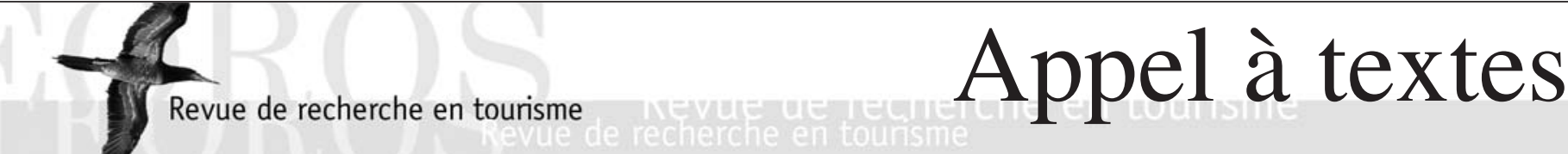

Téoros invite tous les chercheurs et tous les professionnels qui œuvrent dans le domaine du tourisme, ou qui s'intéressent au tourisme sous tous ses aspects, à soumettre des articles de nature analytique à la revue. On peut soumettre un article en l'envoyant à :

Bruno Sarrasin, rédacteur en chef TÉOROS

Département d'études urbaines et touristiques

Université du Québec à Montréal

C.P. 8888, succ. Centre-Ville

Montréal, Québec , Canada

H3C 3 P8

Courriel : teoros@uqam.ca

Tél. : (514) 987-3000, poste 6959

Publiée trois fois I'an depuis 1982, Téoros, revue internationale de recherche en tourisme, se veut un outil intellectuel et professionnel pour les acteurs voués au développement du tourisme. Les problématiques liées aux rapports entre le tourisme et la culture ou entre le tourisme et la société, à la gestion ou à la planifi- cation en tourisme, à des cas particuliers de mise en tourisme ainsi qu'aux questions méthodologiques d'actualité, par exemple, comptent parmi les sujets abordés. Téoros souhaite étendre le champ de ces investigations en lançant un appel élargi à ses lecteurs pour constituer, dans chacun de ses numéros, mais indépendamment de la thématique adoptée, un regroupement de tels articles de nature analytique.

Les textes soumis doivent apporter une contribution scientifique originale, que ce soit par le biais d'information factuelle jusqu'alors inconnue ou par une nouvelle interprétation d'un thème particulier. Téoros vise avant tout le transfert des connaissances; son objectif est donc de promouvoir une meilleure compréhension des phénomènes liés au tourisme auprès d'un lectorat élargi.

Les auteurs doivent faire parvenir un manuscrit présenté selon les règles de la revue, que l'on peut consulter à www.unites.uqam.ca/teoros. Habituellement, un article analytique compte environ
4000 mots et excède rarement 6000 mots, avec deux illustrations; on pourra cependant considérer des textes plus longs ou plus courts.

Les articles peuvent être soumis en anglais ou en francais et doivent être accompagnés d'un résumé de 100 à 200 mots, dans la langue de l'article.

Les auteurs qui n'ont pas accès au site Internet de lo revue peuvent contacter la rédaction pour obtenir copie des règles de présentation qu'ils devront suivre, quant aux références, notamment. Le manuscrit doit être fourni sur support informatique (disquette ou envoi par courriel) en format RTF.

Tous les manuscrits seront évalués par un comité de lecture qui décide des articles qui seront publiés. Le comité peut faire des suggestions ou demander des modifications. La rédaction transmettra l'avis du comité aux auteurs et s'assurera que les modifications demandées seront apportées. La réponse du comité est normalement donnée dans les deux mois suivant la soumission d'un article. 